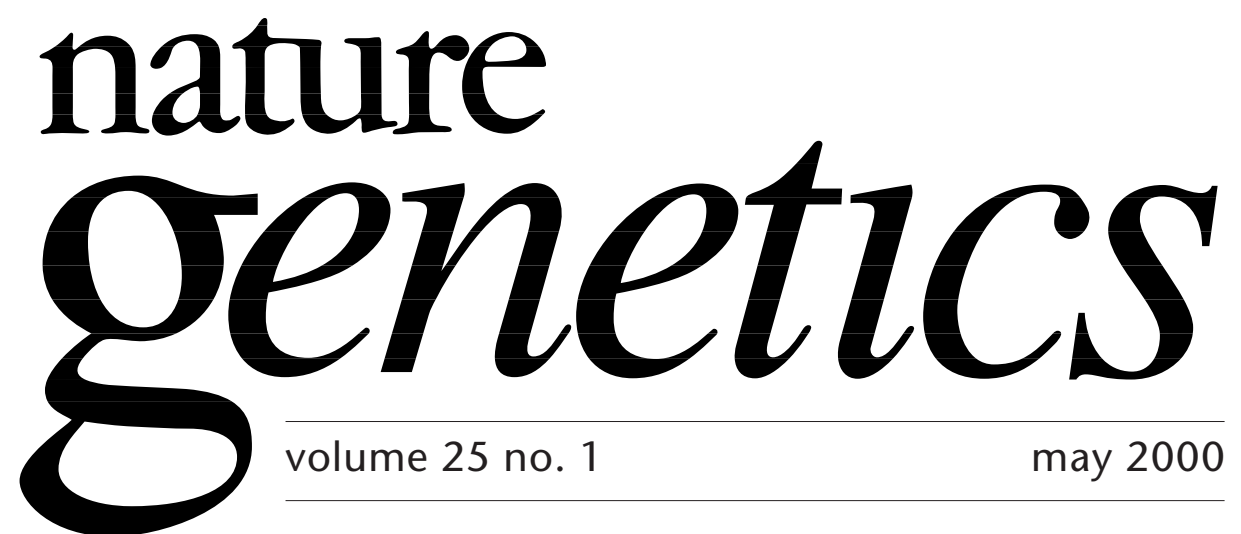

\title{
Sex, genes and women's health
}

Apart from the obvious, what makes men and women different? More specifically, what distinguishes a somatic female from a somatic male cell, and what are the implications for women's health? This was the topic of a recent conference ${ }^{\star}$ held by the Society for Women's Health Research (http://www.womens-health.org). Seventy-five invited participants who couldn't resist the temptation to attend a meeting on 'Sex and Gene Expression' discussed sex-specific differences in genetic composition and gene expression.

David Page (Whitehead Institute) pondered a number of fundamental differences. Male and female cells in all parts of the body experience different hormonal environments. In addition, males have only one X chromosome, which makes them more susceptible to X-linked disorders. Female cells, on the other hand, undergo the biochemical process of $\mathrm{X}$ inactivation, which silences most—but not all-genes of one randomly chosen X chromosome. The subset of genes that escape $\mathrm{X}$ inactivation shows dosage differences between male and female cells, and some degree of reactivation of the inactive $\mathrm{X}$ occurs with age. As a consequence of $\mathrm{X}$ inactivation, females are composed of two epigenetically different cell populations. Page speculated whether this could be a reason for the higher occurrence of autoimmune disorders in females. He also raised the question of imprinted genes on the X chromosome. Phenotypic differences (in social behaviour, for example) between Turner syndrome girls with maternal and paternal X chromosomes, respectively, have been reported, but so far nothing is known about imprinted genes that could underlie these differences. Finally, male cells have-and female cells lack-a Y chromosome, which contains a number of genes, several of them not restricted to the reproductive organs but expressed throughout the male body. RPS4Y, encoding ribosomal protein S4, for example, is quite different from its $\mathrm{X}$ chromosomal counterpart RPS4X, and only male cells contain ribosomes of distinctive 'Y flavour'.

Hormones are powerful regulators of gene expression, and the levels for many of them differ between males and females. As Kathryn Horwitz (University of Colorado) pointed out, women are at a higher risk to develop breast cancer not because they have breasts, but because they have ovaries which produce oestrogen and progesterone. Both hormones bind to nuclear receptors that interact with cofactors to regulate transcription of target genes. Horwitz generated cell lines that express only one of the two progesterone receptors, examined their expression profiles in response to hormone and found distinct sets of target genes. In a related quest to understand hormonal resistance in breast cancer, Donald McDonnell

*First Annual Conference on Sex and Gene Expression, Winston-Salem, North Carolina, March 2-5, 2000. 
(Duke University) focused on the action of oestrogen and drugs that agonize or antagonize its function. The action of many such compounds turns out to be tissue- or cell-type dependent. Tamoxifen, for example, antagonizes oestrogen receptor (ER) function in the breast, but functions as an ER agonist in bone. Structural studies suggest that the ER-oestrogen complex and the ER-tamoxifen complex have different configurations, bind to different nuclear co-factors and regulate distinct sets of target genes. As a result, compounds like tamoxifen-originally thought to function as anti-oestrogens-are now known as selective ER modulators, or SERMs. The system is complex: there are actually two ERs with distinct expression patterns, and little is known about the molecular response to endogenous hormone or to SERMs in the many tissues expressing these receptors. In light of this, McDonnell cautioned that it is difficult to foresee the impact of SERMs, as discussed in the context of breast cancer chemoprevention, or 'replacement hormones' on female health in the long term.

Phyllis Wise (University of Kentucky), a strong advocate of hormone replacement therapy in post-menopausal women, argued that females were not meant to live without oestrogen and presented results from animal studies on oestradiol function in the brain. Epidemiological studies in humans suggested that oestrogen might enhance memory and cognition, decrease the risk and severity of neurodegeneration and protect against ischaemic injury. Wise reported that physiological levels of oestradiol have protective effect in ovarectomized rats with induced brain injury.

Whether the mix of two different cell populations or the female-specific mix of hormones has something to do with the higher incidence of autoimmune disorders in women is not clear, but the skewing is dramatic: $90 \%$ of patients with multiple sclerosis are female, as are $75 \%$ of those suffering from rheumatoid arthritis or systemic lupus erythematosus. Some of the existing animal models for autoimmune disorders reproduce the higher incidence in females and, as illustrated by Denise Faustman (Harvard Medical School), have provided important clues to the molecular basis of autoimmunity. She discussed a finding in the non-obese diabetic mouse model that provides an inroad to the sex-specificity: a single point mutation present in mice of both sexes impairs the transcription of Tap1 and $P s m b 9$, two genes essential for antigen processing and presentation, but only in females. The reasons for the sex-dependent expressivity are still under investigation, but one lesson is clear: the inclusion of female subjects, two and four-legged, and sex-specific analysis is critical. And this applies to research into all diseases that affect women, not just those that are more prevalent in females.

Faustman used the term 'gender' (as did many other participants, particularly those working in the United States) when talking about biological differences between men and women. Once upon a time there was a difference between sex and gender: the former described the biological difference between men and women, the latter relates to the perception of an individual's sexuality. This distinction has apparently vanished, even in the scientific literature. David Haig (Harvard University) examined the frequency of the two terms in scientific citations over the past 20 years. His poster 'Avoiding Sex or the Ascent of Gender: the Sex to Gender Ratio in the Recent Scientific Literature' described not only a dramatic increase in the use of gender, but also that gender frequently substitutes for what used to be called sex. Why blur two distinct entities for which sensible terms exist? Put bluntly by Florence Haseltine (National Institutes of Health and founder of the Society for Women's Health Research), "you can't use 'sex' when you want to get public funding in the United States".

The lines of research discussed at the conference are important and funding for them must not be jeopardized, but one hopes that, at least in grant applications and the scientific literature, correct terminology can be used rather than making gender a politically correct euphemism for sex.

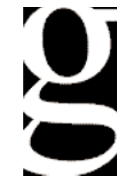

